\title{
Enlargement of the brachial artery: significance of measurement
}

\author{
Toshiaki Otsuka and Ryo Munakata
}

Hypertension Research (2015) 38, 459-460; doi:10.1038/hr.2015.59; published online 16 April 2015

E ndothelial dysfunction is thought to be an Cinitial step in the progression of atherosclerosis. Therefore, in a large number of clinical studies, endothelial function has been evaluated as a marker of cardiovascular risk. ${ }^{1}$ One standard, non-invasive method for evaluating endothelial function is the ultrasonographic measurement of flow-mediated dilatation (FMD) of the brachial artery (brachial FMD). ${ }^{2}$ Brachial FMD is defined as the relative change in brachial artery diameter in response to increased blood flow, usually achieved by deflation of an inflated pneumatic cuff on the forearm, compared with the baseline diameter (that is, expressed as percentage change from baseline). ${ }^{2}$

Abundant evidence suggests that brachial FMD predicts the risk of cardiovascular disease, not only in high-risk patients but also in the general population. ${ }^{3}$ However, there are several limitations to the measurement of brachial FMD. First, this method entails occlusion of the arteries in the forearm for several minutes (usually $5 \mathrm{~min}$ ) as well as multiple measurements of brachial artery diameter (baseline and after deflation of the cuff), which make it a complex and timeconsuming method. Second, measurement of the brachial FMD requires certain examination techniques. In this regard, the coefficient of variation $(\mathrm{CV})$ of brachial artery diameter is acceptable when a highly skilled, dedicated sonographer conducts the image acquisition; however, the CV of FMD per se does not reach an acceptable level, ${ }^{4}$ although a recently developed, semi-automatic device enables us to obtain more stable and reproducible

$\mathrm{T}$ Otsuka is at Department of Hygiene and Public Health, Nippon Medical School, Bunkyo-ku, Tokyo, Japan; R Munakata is at Cardiovascular Center, Nippon Medical School Chiba Hokusoh Hospital, Chiba, Japan E-mail: otsuka@nms.ac.jp results. ${ }^{5}$ The third and probably most important concern is that FMD is reported to be closely and negatively correlated with baseline vessel diameter. ${ }^{6}$ This issue raises the possibility that baseline (that is, non-stress condition) brachial artery diameter has similar clinical significance to that of brachial FMD. In fact, brachial artery diameter is reported to be positively associated with the carotid intima-media thickness, ${ }^{4}$ a surrogate marker of cerebro- and cardiovascular disease. Enlargement of brachial artery diameter is also shown in subjects with coronary artery calcification. ${ }^{4}$ Furthermore, a populationbased cohort study reported that baseline brachial artery diameter is significantly associated with the risk of cardiovascular disease. ${ }^{7}$ The potential usefulness of measuring baseline brachial artery diameter is further supported by Glagov et al., ${ }^{8}$ who indicated that an increased lumen size, known as compensatory arterial remodeling, may be observed in the early stages of atherosclerosis. Taken together, it can be concluded that when further evidence of baseline brachial artery diameter as a cardiovascular risk marker is accumulated, its measurement will be recommended, particularly in cases where measurement of brachial FMD is difficult due to time constraints, such as during routine health examinations.

In this issue, Tomiyama et al. ${ }^{9}$ reported the accuracy and reproducibility of the measurement of brachial artery cross-sectional area using a newly developed, automated oscillometric device. In brief, this device describes the pressure-area curve of the brachial artery using data obtained from the oscillometric measurement. The end-diastolic crosssectional area (estimated cross-sectional area; eA) of the brachial artery is estimated according to the pressure-area curve. The measurement is usually completed within several minutes. The eA is considered virtually identical to the diameter based on the assumption that the arterial lumen is circular. Tomiyama et al. ${ }^{9}$ found that the brachial artery diameter calculated from the eA was closely correlated $(r=0.75, P<0.001)$ with that obtained from the ultrasonographic measurement. The eA on the first and the second measurement days was not significantly different, with good reproducibility (intraclass correlation coefficient $=0.88$ ). The eA was also positively correlated with age $(r=0.56, P=0.026)$. Furthermore, this oscillometric device could detect an increase in the eA after sublingual administration of nitroglycerin. Taken together, these results explicitly indicate the reliability of this oscillometric device for measuring the eA.

The next challenge regarding measurement of the eA by this oscillometric device is to clarify whether it has any clinical significance. In this regard, using the same oscillometric device as in the article by Tomiyama et al., ${ }^{9}$ we previously examined the relationship between the eA and various cardiovascular risk factors in middle-aged men without known cardiovascular disease. ${ }^{10}$ Our study showed that the eA was significantly higher in subjects with obesity, hypertension, dyslipidemia, or impaired fasting glucose/diabetes than in those without these risk factors. In a multiple linear regression analysis, body mass index, age, systolic blood pressure and pulse rate were independent determinants of the eA. The eA showed a weak but significant positive correlation with brachial-ankle pulse wave velocity, one of the standard noninvasive measurements of arterial stiffness. In addition, the eA gradually increased with an upward shift of the Framingham cardiovascular risk category. Although our study 
was a cross-sectional investigation and the causal relationship could not be elucidated, these findings suggest that the eA measured with this oscillometric device may emerge as a significant cardiovascular risk marker.

The most significant advantage of the automated oscillometric device for measuring the $\mathrm{eA}$ is that it does not require any particular expertise. This advantage may partly overcome the limitation of ultrasonographic measurement. In contrast, this device has a considerable methodological limitation. Previous studies reported that a certain percentage of subjects have anomalies or variations of the brachial artery ${ }^{11,12}$ such as double brachial artery, branch variation and vessel tortuosity. These variations may reduce the quality of this oscillometric measurement, and we should therefore pay attention to this limitation when measuring the eA using this oscillometric device.

What do we obtain from this oscillometric measurement? Considering the aforementioned comments, the answer to this question includes the following: (1) enlargement of the brachial artery can be more easily determined than with ultrasonographic measurement, and (2) clinical significance similar to that of brachial FMD may be obtained without any particular expertise. However, further studies are needed to establish the usefulness of this oscillometric measurement in a wide variety of settings in the field of both clinical and preventive cardiology.

\section{CONFLICT OF INTEREST}

TO and RM have received the Health Chronos TM-2771 prototype (A\&D Company, Tokyo, Japan) for the oscillometric measurements.

\section{ACKNOWLEDGEMENTS}

We thank Dr Eitaro Kodani for his valuable suggestions for improving this commentary.

1 Strisciuglio T, De Luca S, Capuano E, Luciano R Niglio T, Trimarco B, Galasso G. Endothelial dysfunc tion: its clinical value and methods of assessment. Cur Atheroscler Rep 2014; 16: 417

2 Tomiyama H, Kohro T, Higashi Y, Takase B, Suzuki T, Ishizu T, Ueda S, Yamazaki T, Furumoto T, Kario K, Inoue $\mathrm{T}$, Koba S, Watanabe $\mathrm{K}$, Takemoto $\mathrm{Y}$, Hano $\mathrm{T}$, Sata M, Ishibashi $Y$, Node K, Maemura K, Ohya $Y$ Furukawa $\mathrm{T}$, Ito $\mathrm{H}$, Yamashina A. A multicenter study design to assess the clinical usefulness of semiautomatic measurement of flow-mediated vasodilatation of the brachial artery. Int Heart J 2012; 53 $170-175$

3 Ras RT, Streppel MT, Draijer R, Zock PL. Flow-mediated dilation and cardiovascular risk prediction: a systematic review with meta-analysis. Int $J$ Cardiol 2013; 168: 344-351.

4 Montalcini T, Gorgone G, Gazzaruso C, Romeo S, Bosco D, Pujia A. Brachial artery diameter measurement: a tool to simplify non-invasive vascular assessment. Nutr Metab Cardiovasc Dis 2012; 22: 8-13.

5 Iguchi T, Takemoto Y, Shimada K, Matsumoto K, Nakanishi K, Otsuka K, Hyodo E, Hirohashi K,
Tahara A, Yoshiyama M. Simultaneous assessment of endothelial function and morphology in the brachial artery using a new semiautomatic ultrasound system. Hypertens Res 2013; 36: 691-697.

6 Atkinson G, Batterham AM. Allometric scaling of diameter change in the original flow-mediated dilation protocol. Atherosclerosis 2013; 226 : 425-427.

7 Yeboah J, Crouse JR, Hsu FC, Burke GL, Herrington DM. Brachial flow-mediated dilation predicts incident cardiovascular events in older adults: the Cardiovascular Health Study. Circulation 2007, 115: 2390-2397.

8 Glagov S, Weisenberg E, Zarins CK, Stankunavicius R, Kolettis GJ. Compensatory enlargement of human atherosclerotic coronary arteries. N Engl J Med 1987; 316: 1371-1375.

9 Tomiyama Y, Yoshinaga K, Fujita S, Ochi N, Inoue M, Nishida M, Aziki K, Horie T, Katoh C, Tamaki N. Accurate quantitative measurements of brachial artery cross-sectional vascular area and vascular volume elastic modulus using automated oscillometric measurements: comparison with brachial artery ultrasound. Hypertens Res 2015; 38: 478-484.

10 Otsuka T, Munakata R, Kato K, Kodani E, Ibuki C, Kusama Y, Seino Y, Kawada T. Oscillometric measurement of brachial artery cross-sectional area and its relationship with cardiovascular risk factors and arterial stiffness in a middle-aged male population. Hypertens Res 2013; 36: 910-915.

11 Rodriguez-Niedenfuhr M, Vazquez T, Nearn L, Ferreira B, Parkin I, Sanudo JR. Variations of the arterial pattern in the upper limb revisited: a morphological and statistical study, with a review of the literature. J Anat 2001; 199: 547-566.

12 Fujii $\mathrm{Y}$, Teragawa $\mathrm{H}$, Soga J, Idei N, Fujimura $\mathrm{N}$, Maruhashi T, Mikami S, Iwamoto Y, Hidaka T, Kihara Y, Chayama K, Noma K, Nakashima A, Goto C, Higashi Y. Flow-mediated vasodilation and anatomical variation of the brachial artery (double brachial artery) in healthy subjects and patients with cardiovascular disease. Circ J 2013; 77 1073-1080. 